\title{
Cerebral atherosclerosis and oxidative stress in Alzheimer's disease
}

\author{
Hernando Rafael* \\ Neurosurgeon Consulting, Hospital Nacional Dos de Mayo. Lima, PERÚ
}

\section{To the edidor}

In our wide experience with omental transplantation to the encephalon in patients with transient ischemic attacks [8] or ischemic infarcts [6,7], and late sequelae in the residual cauda equina and spinal cord [12]; we have observed that neurological improvement is better during the first weeks after the surgery than in the following months or years. These results demonstrated that the function of neurons and/or axons in the residual nervous tissue in ischemia and ischemic penumbra can improve if circulation is restituted through the omentum and, later on, bacause of axonal regeneration,

For these reasons, I found the article by Chen et al. [1] to be of great interest in relation to sporadic Alzheimer's disease (AD). These authors supported the same conclusions by other researchers [3-5] that oxidative stress provoked by transient ischemic/hypoxia injury can cause AD. In other works, in the pathogenesis of $\mathrm{AD}$ there are a cascade of pathophysiologic events characterized by 1) brief or prolonged periods of ischemia, 2) oxidative stress, and finally, 3) a neuronal and axonal death

In my opinion, this cascade of sequential events is initiated in the medial temporal lobes (hippocampal formation, entorhinal area, and the amygdaloid body) and/or subcommissural regions (constituted by cholinergic and neuropeptidic nuclei as well as their fiber

* Correspondence to: Hernando Rafael, M.D., Bélgica 411-BIS, Colonia Portales, 03300 Mexico city, MÉXICO. Tel.: 52555532 9101; Fax: 52555539 5083; E-mail: hrtumi@yahoo.com. bundles, especially medial forebrain bundles) [10,11] by the following reasons. FIRST, nongenetic AD represent about $95 \%$ of all cases and the mean age at onset of the symptoms is 66 years (range 45 to 84 years). SECOND, in all patients with $\mathrm{AD}$, the onset of the symptoms is insidious, course undulating (periods of clinical improvement alternating with those of worsening), and progressive. Clinical findings suggest that the etiologic agent is of vascular origin. THIRD, the early stage (mild degree) of this disease is characterized by 1) impairment of recent memory or 2) behavioral and personality changes,both data as initial symptoms, and with less frequency (about $25 \%$ of cases) associated with 3) olfactory,gustatory or visual deficits.Clinical data suggestives that $\mathrm{AD}$ is initiated in the medial temporal lobes (implicated in the recent memory), subcommissural regions (involved in affective and psychotic changes) and anterior perforated substance (area related with the optic tracts and olfactory striae). FOURTH, in all mild AD patients, computed tomography scans are normal or there exist cerebral atrophy that is normal for the age. Likewise the electroencephalograms are normal, and in some cases, single photon emission computed tomography show hypoperfusion and hypometabolism in the orbitofrontal and/or temporal regions. Consequently, the diagnosis of the early stage (mild AD) of this disease is clinical. FIFTH, tomographic and neurosurgical findings have demonstrated three important observations in the circle of Willis: 1) moderate and/or severe atherosclerosis of the supraclinoid portion of the internal carotid arteries and its terminal branches; 2) a variable number of exsanguinat- 
ed and collapsed anterior perforating arteries originated from the carotid crotch and circle of Willis, and 3) some perforating branches with residual blood flow centripetal to the origin of these vessels. So that there is a reduction in blood flow and impaired nutrient delivery to the parenchymal territory of these colllateral branches.

Therefore, based on our clinical observations, I think that in mild AD patients, the following can occur. FIRST, progressive hypoperfusion into the intraparenchymal territory of the anterior choroidal and anterior perforating arteries due to atherosclerotic plaques located at the mouths of these arterial arterial branches. By contrast, the complete reversal of symptoms in the mild AD patients is due to a revascularization of these affected zones [9,11], and thus, the neuronal injury in ischemia and ischemic penumbra receives through the omentum, an increase in blood flow, oxygen, omental neurotransmitters and neurotrophic factors. SECOND, the ischemic neuronal damage in these medial temporal lobes and/or subcommissural regions can cause molecular and biochemical abnormalities [1,3-5,11], i.e., oxidative stress. THIRD, in late stages (moderate and advanced degrees), neuronal death, axonal degeneration of afferent and efferent projections to the temporal lobes and subcommissural regions, astrogliosis and cerebral atrophy occur, i.e., neurodegeneration.

In summary, I postulate that the earliest stages of $\mathrm{AD}$ are caused by progressive hypoperfusion into the intraparenchymal territory of the anterior choroidal and anterior perforating arteries secondary to stenosis or occlusion by atherosclerotic plaques located at the origin of the collateral branches, and later on, oxidative stress constitutes the major role in the pathogenesis of
AD. Therefore, I agree with de la Torre [2] in to come to the conclusion that $\mathrm{AD}$ is wrongly classified as a neurodegenerative disease.

\section{References}

[1] G-J. Chen, J. Xu, S.A. Lahousse, N.L. Caggiano and S.M. de la monte, Transient hypoxia causes Alzheimer-type molecular and biochemical abnormalities in cortical neurons: potential strategies for neuroprotection, J Alzheimers Dis 5 (2003), 209228.

[2] J.de la Torre, Alzheimer's disease: how does it start? $J$ Alzheimers Dis 4 (2002), 497-512.

[3] U. Dirnagl, Cerebral ischemia: the microcirculation as trigger and target, Prog Brain Res 96 (1993), 49-65.

[4] K. Kitagawa, M. Matsumoto and T. Oda et al., Free radical generation during brief periods of cerebral ischemia may trigger delayed neuronal death, Neuroscience 35 (1990), 551-558.

[5] D. Praticó and N. Delanty, Oxidative injury in diseases of the central nervous system: focus on Alzheimer's disease, Am J Med 109 (2000), 577-585.

[6] H. Rafael, P. Moromizato, M. Espinoza and A. Malpica, Trasplante de epiplón al cerebelo en pacientes con trombosis de la arteria vertebral, Diagnóstico(Perú) 30 (1992), 23-26.

[7] H. Rafael, Omental transplantation, J Neurosurg 89 (1998), 686.

[8] H. Rafael F. Correa, P. Moromizato and M. Espinoza, Hipertensión esencial y trasplante de epiplon al espacio perforado anterior: Caso clínico.Hipertensión (Méx ) 19 (1999), 42-45.

[9] H. Rafael and R. Mego, Omental transposition, Surg Neurol 52 (1999), 539-541.

[10] H. Rafael, E. Fernández and V. Ayulo, Letter to the editor, $J$ Alzheimer Dis 4 (2002), 329-330.

[11] H. Rafael, Cerebral atherosclerosis and mild Alzheimer's disease, Stroke 34 (2003), 106-107.

[12] H. Rafael, Cervical spondylotic myelopathy: surgical results and factors affecting outcome with special reference to age differences, Neurosurgery 53 (2003), 788-789. 